\title{
Nephrogenic systemic fibrosis
}

INSERM

\section{Source}

INSERM. (1999). Orphanet: an online rare disease and orphan drug data base.

Nephrogenic systemic fibrosis. ORPHA:137617

Nephrogenic systemic fibrosis (NSF) is a rare systemic fibrosing condition observed in renally impaired patients and characterized by a hardening and thickening of the skin with fibrotic plaques or papules, pruritus, joint pain and stiffness, muscle weakness, limitation of range of motion, and yellowed eyes. It is generally associated with administration of gadolinium-based magnetic resonance imaging contrast agents (GBCA) in patients with kidney disease. 